RAE-IC, Revista de la Asociación Española de Investigación de la Comunicación vol. 8, núm. 15 (2021), 315-332 ISSN 2341-2690

Recibido el 30 de noviembre de 2020 DOI: https://doi.org/10.24137/raeic.8.15.14 Aceptado el 5 de enero de 2021

\title{
Investigar participando / participar investigando. Relatos de una experiencia etnográfica
}

\author{
Investigate participating / participate investigating. Stories of an \\ ethnographic experience
}

Isidro, María Eugenia

Instituto de Investigaciones Sociales, Territoriales y Educativas (ISTE), Universidad Nacional de Río Cuarto (UNRC) / CONICET mariaeugeniaisidro@gmail.com

Forma de citar este artículo:

Isidro, M. E. (2021). Investigar participando / participar investigando. Relatos de una experiencia etnográfica. RAE-IC, Revista de la Asociación Española de Investigación de la Comunicación, 8(15), 315-332. https://doi.org/10.24137/raeic.8.15.14

\section{Resumen:}

Si bien la etnografía es un enfoque ampliamente usado en las investigaciones en Ciencias Sociales, en este trabajo reflexionamos sobre la particular experiencia de haber realizado un trabajo etnográfico en un movimiento socioterritorial. Realizar una etnografía en un movimiento socioterritorial genera tensiones ya que la participación no solo es esencial para la investigación, sino que también lo es en tanto condición indispensable para formar parte del movimiento. En ese sentido, nos dedicamos a desandar el camino de la investigación describiendo detalladamente cómo se realizó la 
observación participante y se efectuaron las entrevistas; además de analizar cuáles fueron las potencialidades de su uso y profundizaremos en las tensiones a las que se tuvo que hacer frente durante el proceso de investigación poniendo el acento en la reflexividad en tanto herramienta esencial de la investigación social. Lejos de creer que la objetividad se logra a partir de la distancia con los actores estudiados, en este trabajo se intenta dar cuenta que hasta cuando el involucramiento parece ser un obstáculo en la investigación, éste resulta de gran riqueza en el quehacer etnográfico si está acompañado de un profundo trabajo reflexivo.

Palabras clave: movimiento socioterritorial, identidad, etnografía.

\begin{abstract}
:
Although ethnography is a widely used approach in Social Science research, in this work we reflect on the particular experience of having carried out an ethnographic work in a socio-territorial movement. Carrying out an ethnography in a socio-territorial movement generates tensions since participation is not only essential for research, but it is also an indispensable condition to be part of the movement. In this sense, we are dedicated to retracing the path of the investigation, describing in detail how the participant observation was carried out and the interviews were carried out; in addition to analyzing what were the potentialities of its use and we will delve into the tensions that had to be faced during the investigation process placing the accent on reflexivity as an essential tool of social research. Far from believing that objectivity is achieved from the distance with the actors studied, in this work we try to realize that even when involvement seems to be an obstacle in the investigation, this is very rich in ethnographic work if it is accompanied by deep reflective work.
\end{abstract}

Keywords: socio-territorial movement, identity, ethnography. 


\section{INTRODUCCIÓN}

Hacer una etnografía puede implicar grandes desafíos, por eso el propósito de este artículo es compartir algunas reflexiones que surgen de la experiencia de haber realizado un trabajo etnográfico en un movimiento socioterritorial que pueden ser de interés para quien realice estudios similares.

A lo largo del trabajo iremos desandando el camino transitado durante la investigación. En primer lugar, a modo introductorio, realizaremos un breve recorrido por conceptos teóricos centrales en la investigación como son el de "movimientos socioterritoriales" y el de "identidad". Luego, nos dedicamos a desentrañar los pormenores del trabajo etnográfico describiendo detalladamente la implementación de las técnicas empleadas (observación participante y entrevistas), sus potencialidades y riesgos. Al mismo tiempo, profundizamos en las tensiones a las que se tuvo que hacer frente durante el proceso de investigación poniendo el acento en la reflexividad en tanto herramienta esencial del investigador social.

A continuación, los invitamos a recorrer los senderos de esta investigación empezando por algunas consideraciones teóricas para después sí adentrarnos en lo referido a las cuestiones metodológicas que la hicieron posible.

\section{MARCO TEÓRICO}

La noción de movimiento socioterritorial surge a partir de los planteos del geógrafo brasilero Bernardo Mançano Fernandes quien enfatiza la idea de que los movimientos sociales y los socioterritoriales "son un mismo sujeto colectivo o grupo social que se organiza para desarrollar una determinada acción en defensa de sus intereses, en posibles enfrentamientos y conflictos, con el objetivo de la transformación de la realidad" (Fernandes, 2006, p. 8). La diferencia radica en la perspectiva desde la cual se los analiza. En este sentido, cobra vital relevancia el territorio.

Desde la década de 1980, fuertemente vinculado a las ideas de la posmodernidad, comienza a cambiar la concepción del espacio. Deja de ser pensado exclusivamente en su dimensión material y empieza a cobrar fuerza su dimensión simbólica. Se trata de un giro espacial a partir del cual cambia la visión epistemológica desde la que se lo aborda. 
Desde esta perspectiva, el territorio es concebido como un espacio de lucha, contradicción y apropiación, de conformación de subjetividades, lleno de significados culturales y memoria desde donde los movimientos sociales se construyen, articulan físicamente y donde se dan las múltiples relaciones de poder en formas específicas de dominación y resistencia (Haesbaert, 2004; Porto Gonçalves, 2001) y como un espacio de conflictualidades (Fernandes, 2006). Con la finalidad de lograr sus objetivos, los movimientos sociales construyen espacios políticos, se espacializan y promueven cambios en el territorio a partir de procesos de territorialización, desterritorialización y reterritorialización (Fernandes, 2006) en los que construyen su identidad.

Territorialización, desterritorialización y reterritorialización (de ahora en más TDR) son conceptos centrales para el estudio de los movimientos socioterritoriales y, por ende, definirlos es de gran importancia para esta investigación. Como sostiene Rogerio Haesbaert (2004), la forma en la que se los conciba está indefectiblemente ligada a la manera en que se defina al territorio. En el marco de esta investigación, lo entendemos como un espacio social construido a partir de las diversas maneras en que grupos sociales se lo apropian y disputan la construcción del orden. Por ende, lo concebimos ligado al conflicto, como un espacio de "de libertad y dominación, de expropiación y resistencia" (Fernandes, 2006, p. 4). En esta línea, consideramos a la tríada TDR como procesos que marcan diferentes momentos dentro la trayectoria de los movimientos socioterritoriales. La territorialización es el proceso por el cual un grupo social, en este caso un movimiento socioterritorial, se apropia de un espacio y lo transforma en territorio. Se trata del resultado nunca acabado del complejo entramado de relaciones con los otros (aliados o adversarios) y con su entorno que lo condiciona a la hora de plantear sus metas, estrategias y acciones.

La desterritorialización tiene lugar a partir de cambios que se dan en la manera en que un movimiento socioterritorial se apropia del espacio. Pueden ser cambios en las relaciones, en sus metas u objetivos, por transformaciones en el entorno inmediato, por la resolución u profundización del conflicto que lo moviliza, etc. Siempre, luego de un proceso de desterritorialización deviene la reterritorialización, es decir una nueva manera de apropiación del espacio. 
El proceso de desterritorialización y posterior reterritorialización no implica, necesariamente, transformaciones radicales; hay cambios, pero también continuidades. Se tratan de momentos dentro de la trayectoria donde el movimiento va reconfigurando su construcción identitaria.

Apartándonos de concepciones esencialistas que entienden a la identidad como una cualidad intrínseca a las cosas, en esta investigación la concebimos, desde una perspectiva constructivista, como un resultado siempre dinámico, como una construcción permanente que se da a partir de un entramado de relaciones entre actores y con el entorno.

"La identidad es siempre la resultante de un proceso de identificación dentro de una situación relacional, en la medida, también, en que es relativa" (Cuche, 2007, p. 110). En ese sentido, Navarrete sostiene que el concepto de identidad es aporético en tanto que tiene la condición de necesariedad y a la vez de imposibilidad, es decir, es un concepto que es necesario para hablar de algo que caracteriza temporalmente o históricamente a un sujeto o a un campo disciplinario, pero, a la vez, es imposible de representación precisa y definitiva (Navarrete Cazales, 2015).

La identidad es, al mismo tiempo, producto y proceso. Aunque está en continua reconstrucción, tiene cierta permanencia a lo largo del tiempo y es producto de un devenir histórico. La identidad es algo irrepresentable, solo se puede hablar de ella, pero jamás representarla en términos tangibles, definitivos, exhaustivos, ni categóricos. En ese sentido consideramos oportuno utilizar la noción de "identificación" como una manera de adentrarnos a la identidad ya que designa el momento específico de autoreconocimiento con alguien o algo (Navarrete Cazales, 2015), con alguna característica del entorno contextual o del entramado relacional del cual el actor (en este caso colectivo) es parte. Los movimientos sociales se identifican con algo o alguien en momentos específicos, en coyunturas particulares. Esto significa que, a lo largo de su trayectoria, los movimientos sociales van adquiriendo diversas identificaciones que estarían en la base de su identidad, por eso identificarlas y analizarlas permite desandar el proceso de construcción identitario. 
Ahora bien, ¿cómo traducimos esto en términos metodológicos? ¿cómo hacemos para abordar el proceso de construcción identitaria de un movimiento socioterritorial? En el próximo apartado reconstruiremos el recorrido realizado en la investigación, uno de los tantos que se podrían haber seguido.

\section{METOdOLOGÍA}

Consideramos que la mejor manera de abordar el análisis de la identidad de un movimiento socioterritorial es desde la perspectiva etnográfica, método en el cual el investigador participa de la vida cotidiana de un grupo durante un tiempo relativamente extenso, viendo lo que pasa, escuchando lo que se dice, preguntando cosas, o sea recogiendo todo tipo de datos que permitan describir al grupo (Guber, 2012). El trabajo de campo que duró alrededor de dos años, implicó la participación en las múltiples actividades que los integrantes del movimiento llevaban adelante, lo que implicó una instancia fundamental para poder comprender las relaciones que se daban en el entramado interno del propio movimiento como así también las de éste con otros actores y el entorno situacional particular; es decir, la permanencia prolongada en la vida cotidiana del movimiento permitió dilucidar cuáles eran los factores que incidían en el proceso de identificación del movimiento y, al mismo tiempo, dar cuenta de las dinámicas que se producen en las instancias de territorialización / desterritorialización / reterritorialización vivenciadas por el movimiento socioterritorial.

El trabajo etnográfico se hizo en un movimiento socioterritorial de carácter ambiental que surgió en el año 2012 como resistencia al agronegocio en una ciudad mediana del sur de la provincia de Córdoba, fuertemente vinculada a la actividad agrícola. A lo largo de este trabajo nos referiremos al movimiento socioterritorial en cuestión como "Asamblea". Demandas concretas y acciones colectivas frecuentes en defensa del territorio y en contra de todo aquello que perjudique la vida en él; reuniones asamblearias periódicas y toma de decisiones colectivas; trabajo en red con otras organizaciones y la permanencia de sus miembros que posibilita una proyección a largo plazo y la construcción de sentidos y valores compartidos, son elementos que nos habilitan a referirnos a este colectivo como un movimiento social con un fuerte arraigo territorial. 


\subsection{LAS TENSIONES DE LA PARTICIPACIÓN}

Una de las principales características de los movimientos socioterritoriales es su estructura flexible y asamblearia que marca una ruptura con las organizaciones tradicionales como los gremios, sindicatos y partidos (Svampa, 2006). En las asambleas no hay un criterio (por ejemplo, la afiliación) por el cual se pueda entrar o no, el único requisito es la participación, la "presencia", el "estar alli" con continuidad, compromiso y voluntad de generar colectivamente nuevos códigos y sentidos (Giarracca y Petz, 2007). Cada asambleísta tiene la posibilidad de expresar su posición y argumentos ante los temas que son tratados, del mismo modo tiene derecho a votar o, como se da en nuestro caso de análisis, participar de la toma de decisiones a través del consenso resultante del acuerdo colectivo luego de una instancia de deliberación e intercambio de opiniones.

Para los movimientos socioterritoriales, la participación es central. Para la etnografía, también. He aquí el principio de la tensión. Si lo que se pretende es dar cuenta de las relaciones que se entretejen al interior del movimiento, observar vínculos y analizarlos, comprender las decisiones tomadas y acceder a las significaciones otorgadas a las prácticas cotidianas, participar implica mucho más que observar y tomar nota de lo que se ve $u$ oye durante los encuentros con los asambleístas. Participar significaba "ser parte" del movimiento, entiéndase por esto: asistir a las reuniones asamblearias, proponer temas a tratar, opinar sobre los tópicos que se discuten, participar en la toma de decisiones y hacer todo tipo de actividades como salir a la calle a juntar firmas, redactar documentos, asistir a reuniones con funcionarios, entre otras tantas. Es decir, hacer lo mismo que cualquier otro participante.

La situación se complica aún más cuando el investigador se siente identificado con las demandas y objetivos planteados por el movimiento. Ante esto se encuentra en el desafío de redoblar la capacidad reflexiva sobre sus propias prácticas para mantener el equilibrio entre "observación participante" y su "participación militante", tomar la distancia suficiente ante los datos para poder analizarlos y lograr así "una comprensión involucrada, más que una relación externa presentada como objetiva" (Guber, 2018, p. 69). En términos de Fals Borda, podemos hablar de una observación-inserción en la que 
se combina la observación participante con la observación-intervención "para ganar una visión interior completa de las situaciones y los procesos estudiados" lo que conlleva a que la investigadora "se involucre como agente dentro del proceso que estudia, porque ha tomado una posición en favor de determinadas alternativas, aprendiendo así no sólo de la observación que hace sino del trabajo mismo que ejecuta con los sujetos con quienes se identifica" (Fals Borda, 2015, p. 235).

Durante el proceso de investigación, el cuaderno de campo fue el testigo de los momentos de reflexividad. Además de todo lo que se observaba sobre el movimiento en las diferentes instancias de interacción, se anotaban las asociaciones entre teoría y datos que se iban realizando a partir de lo observado y también se iban relevando las apreciaciones personales que surgían del participar, lecturas que indefectiblemente están atravesadas por las propias creencias, experiencias y expectativas. Para el trabajo de reflexividad también fue fundamental el diálogo constante con las directoras de la investigación, el trabajo en equipo resulta un respaldo esencial para un estudio de estas características donde el grado de involucramiento puede poner en riesgo el valor científico de la investigación.

La cuestión de la reflexividad será central en este trabajo etnográfico no sólo por parte de la investigadora sino también por parte de los actores observados, pues la reflexividad se trata, en definitiva, de un proceso de interacción, diferenciación y reciprocidad entre el sujeto cognoscente y los actores observados (Guber, 2013).

\subsection{PARTICIPAR PARA OBSERVAR}

En nuestra investigación, si bien estaba previsto de antemano la realización de observaciones participantes y entrevistas, pensar la manera de implementarlas y el momento oportuno fue todo un desafío.

La observación participante que comenzó allá por inicios de 2016 y que duró alrededor de dos años, fue el eje central del proceso de investigación. Esta técnica consiste en observar sistemática y controladamente todo lo que acontece en torno al investigador $y$, al mismo tiempo, participar en diferentes actividades que involucran a los sujetos observados. 
"Participar" implica que el etnógrafo debe desenvolverse como lo hacen los nativos, comportarse como un miembro más. A partir de la observación se ve a los actores en sus contextos habituales de interacción por lo que se trata de una herramienta útil para contrastar lo que el actor dice hacer y lo que realmente hace (Guber, 2012).

Las reuniones asamblearias y las acciones colectivas realizadas por el movimiento fueron el escenario de las observaciones, como se había proyectado de antemano antes de empezar con el trabajo de campo. Sin embargo, participar activamente de la vida cotidiana del movimiento y tomar responsabilidades acordes al lugar ocupado como participante militante, hizo que pudiera acceder a otro "espacio" de interacción: las redes sociales digitales. Al reconocer la centralidad que éstas tenían tanto en la toma de algunas decisiones como en la organización de actividades, se decidió incorporarlas a la observación.

Durante las reuniones asamblearias el registro de las intervenciones fue lo más exhaustivo y detallado posible, tratando incluso de anotar expresiones textuales de los participantes, el tono de voz con el que lo decía, gestos, mímicas y movimientos del cuerpo. En la medida de lo posible, también se observaron y registraron las reacciones (verbales y no verbales) de los otros interactuantes. La disposición de cada uno de los presentes en el espacio también era una cuestión considerada al momento del registro que consistió en tomar notas en un cuaderno durante todo el encuentro. Nunca se hicieron grabaciones de voz ni videos como así tampoco registros fotográficos durante estas reuniones.

En las observaciones durante las acciones colectivas se consideró el escenario, los actores que participaban, las conductas de los integrantes del movimiento, algunas conversaciones que podrían aportar datos significativos, entre otros aspectos. En estas circunstancias, resultó más complicado tomar notas in situ dado que casi todos los integrantes estaban cumpliendo alguna tarea específica, por eso el registro se realizó una vez terminada la actividad lo más pronto como se podía para no olvidar detalles que podrían resultar relevantes. Cuando la información era demasiada y había riesgos de olvidar algo, la herramienta "notas" del celular se convertía en la mejor opción para escribir algunas ideas que luego serían ampliadas al reconstruir las memorias de la jornada en el cuaderno de notas. 
A medida que transcurría la observación y el registro no sólo de lo que acontecía dentro del movimiento sino también en lo que sucedía con el doble rol de "observadora participante" y de "participante militante" iba tejiendo relaciones, iba sacando conclusiones parciales que parecían explicar al movimiento, sus relaciones y la construcción de su identidad. Al momento de las entrevistas esas categorías que se armaban y rearmaban en el cuaderno de notas fueron puestas en tensión, en discusión.

\subsection{ENTREVISTAS Y DIÁLOGO DE SABERES}

Las entrevistas permiten profundizar en la comprensión de los significados y puntos de vista de los actores sociales. Con la utilización de esta técnica se busca acceder a las significaciones que los actores les otorgan a sus prácticas cotidianas, sus experiencias, a las acciones colectivas, a las relaciones y vínculos entablados tal como las manifiestan con sus propias palabras.

Las observaciones y los datos relevados a partir de ellas fueron las que definieron en qué momento del proceso era oportuno hacer las entrevistas, a quién, cuáles serían los temas que tratar y cómo se desarrollarían. En este último punto fue esencial apelar a la creatividad. El desafío estaba en cómo hacer que los integrantes del movimiento hablen sobre sus prácticas y sus relaciones ante una compañera del grupo que había vivido con ellos la cotidianeidad de los últimos tiempos y que las respuestas no fueran "vos sabés cómo fue, estuviste ahí". Ante este temor latente, se pensó en una dinámica que fuera más allá de la clásica estructura pregunta-respuesta-repregunta y se los invitó a los entrevistados a repensar sus prácticas y repensarse en su lugar dentro del movimiento a partir de otras dinámicas. Apostamos, en este momento de manera más marcada, a propiciar un diálogo de saberes, es decir "un diálogo que se reconoce como un espacio de encuentro y de reconocimientos en la diversidad, que privilegia relaciones de tipo horizontal, al tiempo que valora el disentir y las tensiones que ponen a prueba la creatividad de los participantes para construir propuestas inéditas" (Acosta y Garcés, 2016, p. 21).

Para las entrevistas, en primer lugar, dado que el material con el que se contaba producto de más de un año de observaciones era abundante, se seleccionaron algunos tópicos a ser conversados tratando de hacer un recorte en relación con los objetivos. 
Una vez definido esto, se eligieron los participantes que podrían aportar más a esas cuestiones preestablecidas y se concretaron las entrevistas. Entre una y otra pasó el tiempo necesario para desgrabar, analizar y definir los lineamientos de la próxima.

Los lugares siempre fueron propuestos por los entrevistados. Esto hizo que los escenarios sean de los más variados, pero lo que nunca faltó sobre la mesa, además del grabador de voz con el que se registró cada una de las conversaciones, eran algunos elementos que invitaban al entrevistado a hacer un recorrido por los temas de una manera más didáctica, nos referimos a disparadores como fotos de acciones colectivas o de lugares relevantes en la historia del movimiento; recortes con frases de los entrevistados, de otros compañeros o de funcionarios; pequeños cartones con palabras recurrentemente utilizadas sobre todo en las publicaciones de Facebook; tampoco faltaron hojas en blanco y un fibrón para que el entrevistado pudiera agregar, tachar o corregir lo que creyera conveniente.

Si bien cada entrevista tuvo sus particularidades dado el nivel de confianza y afinidad con el entrevistado, duraron entre una y dos horas. Las conversaciones fueron organizadas en tres partes: en la primera se le preguntaba acerca de los comienzos del entrevistado en el movimiento, sus motivaciones personales y la manera en cómo concebía a la organización; en la segunda parte, se lo invitaba al entrevistado a reflexionar sobre la actualidad del movimiento, acá se ponían en discusión las relaciones establecidas por la investigadora entre datos y entre éstos y las teorías, se reflexionaba acerca de expresiones propias o ajenas que habían sido tomadas de las reuniones asamblearias o de las declaraciones a los medios de comunicación; en tercer y último lugar, se los incentivaba a que pensaran cuáles son los desafíos tanto internos como externos a los que el movimiento se debe enfrentar en la coyuntura actual. Durante las entrevistas se logró que los actores pudieran poner en palabras pensamientos, sentires, reflexiones acerca del movimiento, de ellos en tanto participantes, sus prácticas y relaciones apuntando a su capacidad reflexiva en la que no sólo daban cuenta de su acción y participación sino también que podían reflexionar sobre los significados otorgados y los efectos que éstas tienen en el proceso de identificación. 
Al poner en tensión las categorías que se construyeron durante las observaciones participantes, los aportes de cada entrevistado generaron más preguntas y nuevas relaciones; sacaron a la luz aspectos antes no tenidos en cuenta; hicieron quitar algunas, crear nuevas y renombrar otras. Así, por ejemplo, lo que se había denominado inicialmente "enemigos" a partir de ciertas discusiones que se daban en el marco de las reuniones asamblearias luego de la primera entrevista fue relativizado y pasó a denominarse "adversario" tal como propuso el entrevistado y aceptado por las y los asambleístas entrevistados posteriormente. En otra entrevista, apareció una dimensión intermedia para referirse a las relaciones que se establecen con algunas instituciones estatales (como la Universidad Nacional o la Defensoría del Pueblo, la denominaron "coyunturales". De esta manera, "las voces de los sujetos cobran cada vez mayor protagonismo, se transforman en autores o coautores de los analistas" (Giarracca y Bidaseca, 2007, p. 46), durante el trabajo etnográfico se le dio prioridad a la producción de conocimiento a partir del diálogo con los asambleístas, atendiendo a las diferentes miradas que tienen producto de sus propias experiencias y trayectorias, así los actores involucrados se convierten en actores protagónicos del proceso de construcción del conocimiento.

\section{RESULTADOS}

La implementación de la observación participante y de la entrevista permite describir y analizar la manera en la que el movimiento socioterritorial se identifica en un momento específico de su historia y de la coyuntura social, política y económica de la que es parte. Identificación que depende y es condicionada por factores tales como el entorno situacional, el entramado organizacional, las prácticas y relaciones de territorialización y que la manera en la que se identifica tiene incidencia en los marcos simbólicos desde los cuales interpreta la realidad.

Ahora bien, para poder analizar el proceso de construcción identitaria resulta necesario dimensionar la identificación que el movimiento tiene en un momento específico (en este caso estamos haciendo referencia al periodo 2016-2018) en el marco de su trayectoria. Para eso realizamos un análisis de datos que nos permitió reconocer las diversas maneras en las que el movimiento se identificó en otros momentos de su historia. 
La reconstrucción de la trayectoria del colectivo organizado se logra mediante la información obtenida en las entrevistas, el análisis de fuentes como notas en los medios de comunicación y publicaciones en las redes sociales digitales del movimiento, pero tomamos como punto de partida y fuente principal un documento en el que son los propios asambleístas quienes reconstruyen su historia. Esto fue posible dado a que se trata de un movimiento relativamente "joven" y porque sus integrantes se encargaron de hacer una memoria a lo largo de sus años de existencia. Ese documento fue escrito de manera participativa en tanto implicó que los y las integrantes del movimiento hicieran sus aportes. Si bien fueron dos de los asambleístas quienes le dieron forma y se encargaron de la redacción general, ese documento fue compartido en un grupo cerrado en las redes sociales digitales donde los demás integrantes del movimiento lo leyeron, corrigieron y ampliaron.

Cuando observamos la trayectoria del movimiento podemos dar cuenta que el cambio de algunos de los factores (entorno situacional, el entramado organizacional, las prácticas y relaciones de territorialización) inicia un proceso de desterritorialización y consecuente reterritorialización, es decir se modifica la manera en que el movimiento socioterritorial se autoidentifica y se apropia del espacio. Veamos esto a partir de nuestro estudio de caso. Entre 2016-2018 el movimiento socioterritorial estudiado se sintió interpelado por las políticas neoliberales de ajuste impulsadas por el gobierno nacional y se vio identificado en la resistencia y lucha por los derechos vulnerados. Así comenzó una etapa de apertura, de ampliación de sus demandas y de construcción de amplias redes de resistencia partidos políticos, sindicatos y con otras organizaciones. En este periodo, como lo había sido en sus inicios, prevalecen en su repertorio las acciones colectivas directas ya sea participando activamente en la organización o bien acompañando diferentes manifestaciones que se realizan en la ciudad. Esta participación en múltiples actividades y colectivos genera internamente un desgaste en las relaciones y desemboca en una crisis que los integrantes del movimiento explicitan a finales de 2017 y que se convertirá en un desafío para el comienzo de una nueva etapa en su historia.

Esta sintética descripción nos permite observar cómo diversos factores se conjugan en el proceso de identificación que llevan adelante los movimientos socioterritoriales en un 
entorno situacional específico, cómo intervienen el entramado organizacional que caracteriza las relaciones internas; las prácticas que lleva adelante en el espacio público; las relaciones que mantiene con otros actores sean éstas de alianza o conflicto. Se tratan de factores que hacen a la identificación y a la manera en la que el movimiento se territorializa.

Ahora bien, ¿cómo se resignifica esta identificación en el marco de la identidad? Para responder a esta pregunta creímos oportuno realizar un análisis diacrónico que nos permitiera identificar otras maneras de identificación del movimiento en otros momentos de su historia. Esto fue posible dado a que se trata de un movimiento relativamente nuevo y porque sus integrantes se encargaron de hacer una "memoria" a lo largo de sus años de existencia.

Cuando observamos la trayectoria del movimiento podemos dar cuenta que el cambio de algunos de los factores inicia un proceso de desterritorialización y consecuente reterritorialización, es decir se modifica la manera en que el movimiento socioterritorial se autoidentifica y se apropia del espacio. Tomaremos como ejemplo el proceso de desterritorialización que se produce dentro del movimiento luego de que el intendente de Río Cuarto decretara el rechazo a la instalación de Monsanto en la ciudad a fines de 2013.

Cuando el colectivo emprendió sus primeros pasos había un objetivo claro: evitar que Monsanto se instalara en la ciudad. Apenas se conoció la noticia del inminente arribo de la multinacional vecinos de la ciudad que años antes venían haciendo algunas acciones colectivas esporádicas en contra de hechos puntuales vinculados a lo ambiental, se congregaron y comenzaron a planificar diferentes actividades para visibilizar la situación. La conformación de un grupo estable de participantes permitió definir las demandas y darles continuidad a las actividades. Por entonces la cantidad de participantes creció significativamente. Esto permitió que internamente pudieran organizarse varias comisiones encargadas cada una de tareas diferentes (prensa y difusión, contenido, logística, etc.). Ante la urgencia de la demanda las reuniones asamblearias solían ser semanales y en la toma de decisiones prevalecía la votación a mano alzada, siempre dependiendo de la cantidad de integrantes presentes. 
Así como se multiplicaron los integrantes pasó lo mismo con las acciones colectivas. A la hora de hacer visible los reclamos en la esfera pública, los formatos elegidos por los integrantes de la Asamblea fueron de los más diversos: concentraciones en la Plaza Central y en la Municipalidad, marchas, festivales de música, cortes de ruta, escraches, bicicleteada, entre otros. En este periodo primaron las acciones directas dado que se contaba con un grupo importante de asambleístas que garantizaban la realización de éstas.

En este periodo, la alianza más importante a nivel local la constituye con la Universidad Nacional de Río Cuarto. Esta institución juega un papel central en la resistencia ante la instalación de Monsanto ya que brinda su apoyo a través de una resolución del Consejo Superior en la que manifiesta el rotundo desacuerdo con la instalación de la multinacional en la ciudad. Para la Asamblea, este respaldo público de una de las instituciones más importantes de la ciudad y la región le otorgó "gran legitimidad" a su reclamo. Otro hecho fundamental que marcó la estrecha relación Universidad-Asamblea fue la creación del Observatorio de Conflictos Socioambientales a comienzos del 2012 con el que el movimiento llevó adelante algunas actividades. Para los movimientos sociales que resisten al neoextractivismo el respaldo que brindan instituciones públicas como es en este caso una universidad nacional es un apoyo fundamental.

Ahora bien, ¿qué pasó después del "No a Monsanto"?

Con el objetivo de evitar la instalación de Monsanto cumplido, el número de integrantes de la Asamblea disminuyó considerablemente. Empezó así el momento de la reestructuración, de plantarse nuevas metas, de reorganizar el colectivo y redefinir las acciones a seguir.

Este periodo en la trayectoria de la Asamblea se caracterizó por la realización de actividades de formación principalmente en temas vinculados a lo ambiental tanto al interior del movimiento como así también para la sociedad en general. Por eso las acciones que organiza el movimiento en esta etapa son actividades de índole cultural como presentaciones de libros, proyección de películas y charlas debates con especialistas en la temática. 
En lo referido a las articulaciones con otras organizaciones e instituciones, en 2015, la Asamblea pierde a un aliado institucional relevante en su lucha ya que el Observatorio de Conflictos Socioambientales de la Universidad Nacional de Río Cuarto deja de funcionar. Sin embargo, en este periodo el movimiento socioambiental consolida su participación en la UAC (Unión de Asambleas Ciudadanas), un espacio de intercambio, discusión y acción que tiene como propósito articular y potenciar las diferentes luchas que emergieron en el país para repudiar el avance de emprendimientos neoextractivistas, logrando la construcción de redes de lucha y resistencia.

Así como en la etapa anterior el movimiento se identifica principalmente con la resistencia, en este período se identifica con la acción desde la proposición. La campaña por un Río Cuarto Agroecológico, si bien fue impulsada desde 2013, en esta nueva etapa va a adquirir centralidad en la cotidianidad del movimiento y las acciones colectivas van a rondar en la juntada de firmas en diferentes puntos de la ciudad para que el proyecto pueda ser presentando en el Concejo Deliberante como iniciativa popular.

\section{CONCLUSIONES}

La experiencia de investigación desarrollada en este trabajo muestra una manera de aproximarnos a la construcción identitaria de un movimiento socioterritorial. Desentrañar los modos en las que se identifica el movimiento a lo largo de su trayectoria considerando las diferentes etapas por las que pasa a partir de los procesos territorialización que recorre, permite abordar la identidad construida, hacerla de alguna manera aprehensible, aunque no se pueda hablar de ella en términos definitivos.

El enfoque etnográfico nos permite obtener la flexibilidad que necesitamos para poder abordar las maneras en las que colectivo se identifica en un momento particular de su trayectoria. Sin embargo, también pudimos observar en el trabajo que cuando el nivel de involucramiento del investigador con los actores que analiza es demasiado alto resulta complejo salir del campo y tomar distancia necesaria para analizar los datos. Es ahí cuando la reflexividad cobra un papel primordial y se vuelve fundamental para hacer de ésta una investigación científica. 
A lo largo del trabajo pudimos observar no sólo las tensiones que se generaron en torno al rol de la investigadora y la importancia de la reflexividad durante el trabajo de campo sino también cómo se utilizaron algunas herramientas para potenciar la capacidad reflexiva de los propios actores (por ejemplo, retomando durante las entrevistas expresiones, propias o de otros asambleístas, que sirvieron como disparadores o dándoles la posibilidad que intervengan en las categorías que la investigadora había construido hasta ese momento), lo que permitió retroalimentar y enriquecer el trabajo de campo a partir de la coproducción de datos entre actor observado-investigador observador.

Al ser parte del mundo que estudiamos, la reflexividad es esencial en la tarea del investigador social. Entendida como la capacidad que tienen los actores sociales para volver sobre sus propias prácticas y poner en palabras sus percepciones acerca de ellas, la reflexividad cobra relevancia en las Ciencias Sociales asociada a la "etnografía" y a las metodologías de corte cualitativo.

\section{REFERENCIAS BIBLIOGRÁFICAS}

Acosta, G. y A. Garcés (2016). Editorial. El diálogo de saberes en comunicación: reconfiguraciones de la formación y de la investigación. Revista Anagramas, 14, 17-23. Recuperado de http://www.scielo.org.co/pdf/angr/v15n29/v15n29a01.pdf

Arfuch, L. (2005). Problemáticas de la identidad. En L. Arfuch (Comp.), Identidades, sujetos y subjetividades (pp. 21-43). Buenos Aires: Prometeo.

Fernandes, B. (2006). Movimientos socioterritoriales y movimientos socioespaciales. Contribución teórica para una lectura geográfica de los movimientos sociales. Acaoterra.org. Recuperado de http://www.acaoterra.org/IMG/pdf/Movimientossocioterritoriales-y-movimientos-socioespaciales.pdf

Giarracca, N. y Bidaseca, K. (2007). Ensamblando las voces: los actores en el texto sociológico. En A. Kornblit (Coord.), Metodologías cualitativas en ciencias sociales. Modelos y procedimientos de análisis (pp. 35-46). Buenos Aires: Editorial Biblos. 
Giarracca, N. y Petz, I. (2007). La Asamblea de Gualeguaychú: su lógica La Asamblea de Gualeguaychú: su lógica de nuevo movimiento social y el sentido de nuevo movimiento social y el sentido binacional "artiguista" de sus acciones binacional "artiguista" de sus acciones. Realidad Económica, 226, 101-126.

Guber, R. (2018). "Volando rasantes"...etnográficamente hablando. Cuando la reflexividad de los sujetos sociales irrumpe en la reflexividad metodológica y narrativa del investigador. En J. Piovani y L. Muñiz Terra (Coord.), ¿Condenados a la reflexividad? Apuntes para repensar el proceso de investigación social (pp. 52-72). Buenos Aires: CLACSO, Biblos.

Guber, R. (2013). El salvaje metropolitano. Buenos Aires: Paidós.

Guber, R. (2012). La etnografía: Método, campo y reflexividad. Buenos Aires: Siglo veintiuno editores.

Svampa, M. (2006). Movimientos sociales y nuevo escenario regional: inflexiones del paradigma neoliberal en América Latina. Cuadernos de Socio-Historia, 19/20, 141-155.

Zibechi, R. (2003). Los movimientos sociales latinoamericanos: tendencias y desafíos. OSAL: Observatorio Social de América Latina. No. 9. Buenos Aires: CLACSO. 